\title{
Diagnostic value of an immunochromatographic test over clinical predictors for tuberculosis in HIV patients
}

This article was published in the following Dove Press journal:

Clinical Epidemiology

9 September 201 I

Number of times this article has been viewed

\author{
Sirisak Nanta ${ }^{1,2}$ \\ Patcharee Kantipong ${ }^{3}$ \\ Panita Pathipvanich ${ }^{4}$ \\ Chidchanok Ruengorn ${ }^{5}$ \\ Chamaiporn Tawichasri' \\ Jayanton Patumanond' \\ 'Clinical Epidemiology Program, \\ Faculty of Medicine, Chiang Mai \\ University, Chiang Mai, Thailand; \\ ${ }^{2}$ Maesai District Hospital, Maesai, \\ Chiang Rai, Thailand; ${ }^{3}$ Chiang Rai \\ Regional Hospital, Chiang Rai, \\ Thailand; ' ${ }^{4}$ ampang Regional Hospital, \\ Lampang, Thailand; ${ }^{5}$ Department \\ of Pharmaceutical Care, Faculty of \\ Pharmacy, Chiang Mai University, \\ Chiang Mai, Thailand
}

Correspondence: Jayanton Patumanond Clinical Epidemiology Unit,

Faculty of Medicine,

Chiang Mai University,

Chiang Mai 50200, Thailand

Tel +66 53946306

Fax +66 53945476

Email j.patumanond@yahoo.com
Purpose: The value of an immunochromatographic test for tuberculosis (ICT-TB) combined with clinical predictors has yet to be evaluated in Thailand. This study aimed to assess any additional diagnostic value of an ICT-TB test over that of clinical predictors in a group of human immunodeficiency virus (HIV) patients as well as in subgroups of HIV patients classified by clinical risk scores.

Patients and methods: An extended cross-sectional study was conducted at a community hospital in Chiang Rai and a general hospital in Lampang. HIV patients registered between April 2009 and May 2010 were screened by a locally made ICT-TB test, including 38, 16, and $6 \mathrm{kD}$ Microbacterium tuberculosis antigens, as well as by routine evaluations for TB diagnosis. Demographic data, medical history, signs, and symptoms were recorded. Participants were followed up for 2 months for final ascertainment of TB diagnosis.

Results: Of 206 patients, 37 (18\%) had TB. Four clinical predictors were identified: low body mass index $\left(<19 \mathrm{~kg} / \mathrm{m}^{2}\right)$, prolonged cough (duration $>2$ weeks), shaking chills ( $\geq 1$ week), and no use of antiretrovirals. The area under the receiver operating curve was $90.2 \%$; adding the ICT-TB test result increased the area nonsignificantly to $91.6 \%(P=0.40)$. When patients were categorized by risk scores derived from selected clinical predictors into low (scores $\leq 7$ ) and high (scores $>7$ ) TB risk groups, a positive ICT-TB test increased the positive predictive value nonsignificantly in the low risk group (from $12.5 \%$ to $27.3 \%, P=0.17$ ) and the high risk group (from $78.6 \%$ to $80.8 \%, P=0.73$ ).

Conclusion: In this study setting, the ICT-TB test did not enhance TB diagnosis over the four clinical predictors in the overall group or any subgroups of HIV patients classified by clinical risk scores.

Keywords: diagnostic test, signs, symptoms, TB

\section{Introduction}

Human immunodeficiency virus (HIV) and tuberculosis (TB) co-infection constitutes a high-burden disease in Thailand, where 12,890 individuals died from TB in 2009 and where HIV accounts for $17 \%$ of TB cases, the highest figure in the south-east Asian region. ${ }^{1}$ Early case identification followed by adequate treatment is the cornerstone of TB control programs. Thailand's rank of 18th among 22 high-burden countries for TB is partly attributed to inadequate case evaluation and treatment default, which also result in increased deaths and contribute to underperformance on the World Health Organization (WHO) target for treatment success (81\% versus $85 \%){ }^{1}$

Efforts to improve TB diagnosis by new technology have commonly faced the challenges of high equipment cost, lack of skilled technicians, and excessive 
turnaround time. ${ }^{2}$ Rapid immunochromatographic test (ICT) technology may be the exception to this rule: when used as a serodiagnostic test for TB antibodies (ICT-TB), this technology is affordable, simple and safe to use, and produces results within 25 minutes. ${ }^{3}$ However, in an HIVinfected population, several antigens may be recognized by the antibodies, thereby limiting the diagnostic validity of such tests. ${ }^{4}$ Previous studies have reported a wide range of sensitivity of ICT-TB tests in HIV patients (from $0 \%$ to $80 \%$ ); specificity was also very variable, ranging from $39 \%$ to $97.4 \% .^{4-8}$ A landmark study, conducted by the WHO Special Programme for Research and Training in Tropical Diseases in 2008, showed poor performance of 19 commercial rapid diagnostic tests in HIV patients, with sensitivity reduced by an average of $22 \%-43 \%$ compared with that in HIV-negative patients. ${ }^{9}$ In Thai settings, only two diagnostic validity studies, on the use of lipoarabinomannan and 38-kD recombinant antigens using pleural effusion and serum, have been reported., ${ }^{4,5}$

In an intensified effort to find cases of active TB in Thailand, a general screening tool, which disregards HIV status, is currently being used; this tool consists of simple questions relating to signs and symptoms of TB. From a recent review, the best predictors for TB diagnosis in HIV patients living in resource-constrained settings are presence of cough (for any duration), fever, night sweats, or weight loss. ${ }^{10}$ Other signs, symptoms, and simple laboratory tests proposed by other studies are fatigue, ${ }^{11,12}$ shaking chills, ${ }^{12}$ loss of appetite, ${ }^{12}$ difficulty in breathing, ${ }^{12}$ chest pain, ${ }^{12}$ abdominal pain, ${ }^{12}$ nausea/vomiting, ${ }^{12}$ body mass index (BMI), ${ }^{11,12}$ lymphadenopathy, ${ }^{11,12}$ hemoglobin level of $<10 \mathrm{~g} / \mathrm{dL},{ }^{12}$ tuberculin skin test positivity, ${ }^{12}$ hospitalization upon registration, ${ }^{12}$ lack of antiretroviral (ARV) therapy, ${ }^{11,12}$ chest radiograph abnormalities, ${ }^{11,13,14}$ and CD4 cell count. ${ }^{11,14}$ However, the proposed combinations of predictors, as well as the performance of such predictor combinations, varies between studies. The validity of such existing screening tools is unknown in the setting of Thailand; furthermore, the value of an ICT-TB test used in combination with clinical predictors has yet to be evaluated in Thailand.

Therefore, the objective of this study was to identify clinical characteristics, including demographics, signs, and symptoms that may be used to predict the presence of active $\mathrm{TB}$, as well as to evaluate the diagnostic value of ICT-TB testing over such clinical predictors in subgroups of HIV patients stratified by clinical risk scores.

\section{Materials and methods}

\section{Study settings and population}

The study protocol was approved by two institutional review boards: The Faculty of Medicine, Chiang Mai University and Lampang Regional Hospital.

All HIV patients aged 18 years or older seeking medical care or reporting for annual checkup at either a TB clinic or an ARV clinic between April 2009 and May 2010, regardless of $\mathrm{TB}$ signs and symptoms, were eligible for the study. Outpatient data, including clinical signs and symptoms, and blood samples were collected on the first day. Data from those admitted as inpatients were collected in the wards.

Study settings included Maesai District Hospital, a community hospital in Chiang Rai, and Lampang Hospital, a general hospital in Lampang, Thailand. Patients who were receiving TB treatment or isoniazid prophylaxis treatment (IPT) at the time of or within 1 year prior to recruitment were excluded.

\section{Data collection}

The attending physician or trained nursing staff interviewed participants and recorded data in a case record form. Data collected included patient demographics and clinical data, comprising medical history, history of TB diagnosis, history of TB contact, and ARV prescription. Data regarding duration of signs and symptoms were also collected; these included cough, cough with sputum, hemoptysis, fever, diarrhea, weight loss, night sweats, dyspnea, chest pain, nausea or vomiting, abdominal pain, loss of appetite, fatigue, shaking chills, and size of lymphadenopathy. Laboratory results at recruitment, such as abnormal chest radiograph, complete blood count, and CD4 cell count, were also collected.

\section{Index and reference tests ICT-TB}

As the index test, a locally produced ICT-TB test containing a membrane strip pre-coated with colloid gold-conjugated recombinant Microbacterium tuberculosis antigens (38, 16, and $6 \mathrm{kD})$ was used. In this test, the patient's sample migrates chromatographically along the membrane to the test region, where a visible line forms if an antigenantibody-antigen-gold particle complex forms (positive result). The absence of a visible color band within the test window indicates a negative result. The test was interpreted as invalid if the reference and the control bands did not appear. $^{15}$ 
Blood serum specimens, approximately $100 \mu \mathrm{L}$, were obtained on the day of recruitment by research staff who had received training in the implementation of the ICT-TB test. All patients were tested with the ICT-TB on the day of enrolment or not later than 3 days after enrolment. Results were interpreted within 20 minutes by three interpreters blinded to each other's findings and to the patient's clinical information. When at least two readers indicated a positive result, the result was recorded as "positive." Indeterminate results were excluded from the analysis of the ICT-TB test performance.

\section{Reference tests}

Criteria for a definitive TB diagnosis were defined as smear positivity, positive culture of sputum or other specimens, positive clinical and radiological evaluation, response to TB treatment, or histological characteristics indicative of TB in biopsy specimens, as is the standard clinical practice. Only for registered TB cases, sputum or other specimens were sent for M. tuberculosis culture. All patients were followed up for 2 months to ascertain TB diagnosis.

\section{Statistical analysis}

Predictors of TB were selected using a two-stage procedure. First, predictors with a $P$-value of $<0.05$ were selected by univariate logistic regression. Second, using multivariate logistic regression with a forward method of model selection, ${ }^{16}$ predictors of TB in the final model were selected not only from statistical significance but also from clinical significance. Missing predictor values were dealt with using a multiple imputation method.

Models with/without the ICT-TB test were compared by adding the ICT-TB test results into the clinical predictor model. To assess whether the model prediction was improved by the ICT-TB, two methods were used: the area under receiver operating characteristic curve (AuROC) method, and the net reclassification improvement (NRI) method. ${ }^{17}$ Models with and without ICT-TB were validated and assigned classical diagnostic values.

For the scoring scheme, each variable from the final model was weighted by its coefficient and transformed to an item score. The cutoff points of total scores, determined by statistical significance and the yield of prediction, were used to classify HIV patients into the low- or high-risk groups. The positive predictive value (PPV) of the ICT-TB test for each category was compared with the prior probability (prevalence) by the Fisher's exact probability test. Statistical significance level was set at 0.05 for type I error.
The number of patients required in the study was estimated based on previous information of an odds ratio of 9.6 and $16.9 \%$ prevalence in the non-TB group. ${ }^{15}$ Thus, to achieve $90 \%$ power and a significant finding at alpha $=0.05$, 18 active TB patients were required. Given the prevalence of active TB (17\%) in the study population, ${ }^{18} 106 \mathrm{HIV}$ patients were needed; in order to compensate for $20 \%$ missing data, the final minimum number of HIV patients needed was 128 .

\section{Results}

Of 213 HIV patients eligible for the study, seven (3.3\%) were excluded because of ongoing TB and IPT treatment at the time of enrolment. The final analysis included 206 HIV patients, all of whom underwent the ICT-TB tests. Of these, 37 (18\%) were diagnosed with active TB, including $33(94.3 \%)$ with pulmonary TB and four (5.7\%) with extrapulmonary TB cases (Figure 1).

Using univariate logistic regression, the following patient characteristics, signs, and symptoms were significant: BMI, history of contact, CD4 count level, ARV prescribed, cough, fever, weight loss, dyspnea, chest pain, loss of appetite, fatigue, and shaking chills (Tables 1 and 2).

The predictors that remained in the final model were cough, shaking chills, BMI, and ARV prescribed, with CD4 clustered in the model (Table 3 ). Although those with a positive ICT-TB test result were 5.05 times more likely to have active TB (95\% confidence interval, 0.66-38.48) compared with those who tested negative, the ICT-TB test itself did not add any statistical significance to the final model (Table 3 ).

These predictors explained $90.2 \%$ of the probability (AuROC) of active TB. When the ICT-TB test result was added in the model, it increased the probability of active TB to $91.6 \%$; this increase was not statistically significant $(P=0.40)$ (Figure 2). This nonimprovement of the model by the ICT-TB test result was also confirmed by the NRI analysis $(P=0.51)$. Adding the ICT-TB test result did not enhance any of the diagnostic values in the validation of the model (without ICT-TB versus with ICT-TB): sensitivity, 48.0\% versus $52.0 \%$; specificity, $95.3 \%$ versus $94.7 \%$; PPV, 63.2\% versus $61.9 \%$; and NPV, $91.7 \%$ versus $92.2 \%$. Both models had equal values for correct classification (88.6\%).

This study further investigated whether the ICT-TB would enhance the diagnosis in subgroups of individuals with low versus high risk of having TB. The subgroups were categorized by a risk score system developed from each selected predictor, weighted by the coefficient from the final 


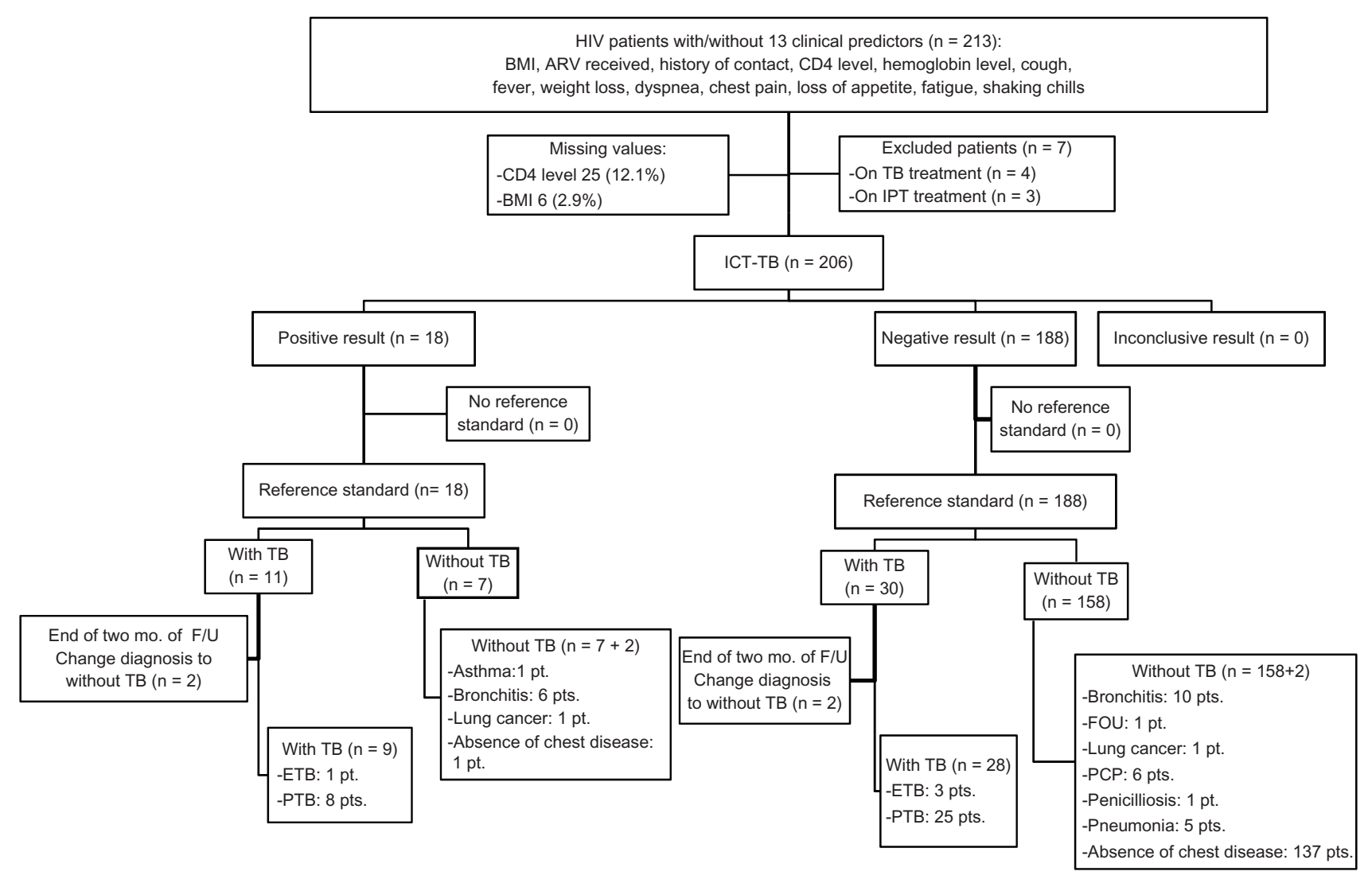

Figure I Study flow diagram for TB diagnosis by clinical predictors combined with ICT-TB in HIV patients.

Abbreviations: ARV, antiretroviral; BMI, body mass index; ETB, extrapulmonary TB; FOU, fever of unknown origin; F/U, follow-up; HIV, human immunodeficiency virus; ICT-TB, immunochromatographic test for tuberculosis; IPT, isoniazid prophylaxis treatment; mo, months; PCP, Pneumocystis carinii pneumonia; pt, patient; PTB, pulmonary TB; TB, tuberculosis.

Table I Characteristics of HIV patients with $(n=37)$ or without TB $(n=169)$

\begin{tabular}{|c|c|c|c|c|}
\hline Characteristics & With TB & Without TB & $\begin{array}{l}\text { Crude OR } \\
(95 \% \mathrm{Cl})\end{array}$ & $P$-value \\
\hline Male & I 8 (48.6) & $82(48.5)$ & $1.00(0.49-2.03)$ & 0.989 \\
\hline \multicolumn{5}{|l|}{ Age, years } \\
\hline Mean \pm SD & $38.4 \pm 12.1$ & $39.9 \pm 9.6$ & $0.99(0.95-1.02)$ & 0.414 \\
\hline \multicolumn{5}{|l|}{$\mathrm{BMI}, \mathrm{kg} / \mathrm{m}^{2}$} \\
\hline$<19$ & $20(58.8)$ & $42(25.6)$ & $4.15(1.92-8.94)$ & $<0.001$ \\
\hline ARVs not prescribed & $28(75.7)$ & $27(16.0)$ & $16.36(6.95-38.52)$ & $<0.001$ \\
\hline History of OI & $4(10.8)$ & $38(22.5)$ & $0.42(0.14-1.25)$ & 0.120 \\
\hline History of TB diagnosis & $6(16.2)$ & $17(10.1)$ & $1.73(0.63-4.47)$ & 0.286 \\
\hline History of TB contact & $5(13.5)$ & $5(3.0)$ & $5.12(1.40-18.73)$ & 0.013 \\
\hline \multicolumn{5}{|l|}{ CD4 cell count, cells/ $\mu \mathrm{L}$} \\
\hline$<200$ & $19(70.4)$ & $33(21.4)$ & $8.71(3.50-21.66)$ & $<0.001$ \\
\hline \multicolumn{5}{|l|}{ Hemoglobin, g/dL } \\
\hline$<10$ & $15(46.9)$ & $18(11.3)$ & $6.96(2.98-16.286)$ & $<0.001$ \\
\hline \multicolumn{5}{|l|}{ WBC count, $1000 / \mathrm{dL}$} \\
\hline Median (range) & $7.3(I-23.2)$ & $6.0(I-24)$ & $1.10(0.99-1.23)$ & 0.064 \\
\hline \multicolumn{5}{|l|}{ Hematocrit, \% } \\
\hline Median (range) & $31.8(13.7-45.7)$ & $37.7(8.5-49.9)$ & $0.88(0.82-0.94)$ & $<0.001$ \\
\hline \multicolumn{5}{|l|}{ Platelet count, $1000 / \mathrm{mL}$} \\
\hline Median (range) & $298.0(69-6 \mid 2)$ & $317.0(28-912)$ & $1.00(0.99-1.00)$ & 0.774 \\
\hline
\end{tabular}

Notes: Numbers are $\mathrm{n}(\%)$, mean $\pm \mathrm{SD}$, or median (range).

Abbreviations: BMI, body mass index; Cl, confidence interval; HIV, human immunodeficiency virus; OI, opportunistic infection; OR, odds ratio; SD, standard deviation; TB, tuberculosis; WBC, white blood cell. 
Table 2 Signs and symptoms of HIV patients with $(n=37)$ or without TB $(n=169)$

\begin{tabular}{|c|c|c|c|c|}
\hline Signs and symptoms & With TB & Without TB & $\begin{array}{l}\text { Crude OR } \\
(95 \% \mathrm{Cl}) \\
\end{array}$ & $P$-value \\
\hline \multicolumn{5}{|l|}{ Cough } \\
\hline$\leq 2$ weeks & $20(54.1)$ & $159(94.1)$ & 1.0 & \\
\hline$>2$ weeks & $17(45.9)$ & $10(5.9)$ & I3.52 (5.44-33.54) & $<0.001$ \\
\hline \multicolumn{5}{|l|}{ Cough with sputum } \\
\hline$\leq 2$ weeks & $25(67.6)$ & I $64(97.0)$ & 1.0 & \\
\hline$>2$ weeks & $12(32.4)$ & $5(3.0)$ & $15.74(5.1 \mid-48.49)$ & $<0.001$ \\
\hline \multicolumn{5}{|l|}{ Hemoptysis } \\
\hline$\leq$ I week & $35(94.6)$ & $168(99.4)$ & 1.0 & \\
\hline$>$ I week & $2(5.4)$ & $\mathrm{I}(0.6)$ & $9.49(0.84-107.53)$ & 0.069 \\
\hline \multicolumn{5}{|l|}{ Fever } \\
\hline$<$ I week & $30(81.1)$ & $156(92.3)$ & 1.0 & \\
\hline$\geq$ I week & $7(18.9)$ & I3 (7.7) & $2.80(1.03-6.00)$ & 0.043 \\
\hline \multicolumn{5}{|l|}{ Diarrhea } \\
\hline$<$ I week & $35(94.6)$ & $167(98.8)$ & 1.0 & \\
\hline$\geq$ I week & $2(5.4)$ & $2(1.2)$ & $4.77(0.65-35.03)$ & 0.124 \\
\hline \multicolumn{5}{|l|}{ Weight loss } \\
\hline$\leq$ I week & $19(52.8)$ & $150(89.8)$ & 1.0 & \\
\hline I-2 weeks & $4(I I . I)$ & $4(2.4)$ & $7.60(1.76-32.80)$ & 0.007 \\
\hline$\geq 2$ weeks & $13(36.1)$ & $13(7.8)$ & $7.60(3.09-18.68)$ & $<0.001$ \\
\hline \multicolumn{5}{|l|}{ Night sweats } \\
\hline$<$ I week & $34(91.9)$ & $162(96.4)$ & 1.0 & \\
\hline$\geq$ I week & $3(8.1)$ & $6(3.6)$ & $2.40(0.57-10.06)$ & 0.232 \\
\hline \multicolumn{5}{|l|}{ Dyspnea } \\
\hline$\leq$ I week & $30(81.1)$ & $159(94.1)$ & 1.0 & \\
\hline$>$ I week & $7(18.9)$ & $10(5.9)$ & $3.7 I(\mid .3 I-10.5 I)$ & 0.014 \\
\hline \multicolumn{5}{|l|}{ Chest pain } \\
\hline$\leq$ I week & $25(67.6)$ & $159(94.1)$ & 1.0 & \\
\hline$>$ I week & $12(32.4)$ & $10(5.9)$ & $7.63(2.98-19.52)$ & $<0.001$ \\
\hline \multicolumn{5}{|l|}{ Nausea/vomiting } \\
\hline$\leq$ I week & $36(97.3)$ & 168 (99.4) & 1.0 & \\
\hline$>$ I week & I (2.7) & I (0.6) & $4.67(0.29-76.36)$ & 0.280 \\
\hline \multicolumn{5}{|l|}{ Abdominal pain } \\
\hline$\leq 2$ weeks & $36(97.3)$ & $166(98.2)$ & 1.0 & \\
\hline$>2$ weeks & I (2.7) & $3(1.8)$ & $1.19(0.13-11.00)$ & 0.877 \\
\hline \multicolumn{5}{|l|}{ Loss of appetite } \\
\hline$\leq$ I week & $29(78.4)$ & I55 (9I.7) & 1.0 & \\
\hline$>$ I week & $8(21.6)$ & $14(8.3)$ & $3.05(1.18-7.94)$ & 0.022 \\
\hline \multicolumn{5}{|l|}{ Fatigue } \\
\hline$\leq$ I week & $25(67.6)$ & I53 (9I.I) & 1.0 & \\
\hline$>$ I week & $12(32.4)$ & I5 (8.9) & $4.93(2.07-11.75)$ & $<0.001$ \\
\hline \multicolumn{5}{|l|}{ Shaking chills } \\
\hline$<$ I week & $31(83.8)$ & $164(97.0)$ & 1.0 & \\
\hline$\geq$ I week & $6(16.2)$ & $5(3.0)$ & $6.35(1.82-22.10)$ & $<0.001$ \\
\hline \multicolumn{5}{|l|}{ Lymphadenopathy } \\
\hline No & $35(94.6)$ & $169(100.0)$ & - & $0.032^{\mathrm{a}}$ \\
\hline Yes & $2(5.4)$ & $0(0.0)$ & NA & \\
\hline
\end{tabular}

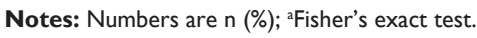

Abbreviations: $\mathrm{Cl}$, confidence interval; HIV, human immunodeficiency virus; NA, not applicable; OR, odds ratio; TB, tuberculosis.

model, and transformed by dividing the value by the smallest coefficient (1.5 for cough and BMI). The value derived was rounded up to the nearest whole number to assign a final score; scores ranged from 0 to 11 (Table 4).
When HIV patients were classified into low TB risk ( scores $\leq 7$ ) and high TB risk (scores $>7$ ) groups, the PPV of ICT-TB test (post-test probability) increased insignificantly from prevalence (pre-test probability) in both the low risk 
Table 3 Multivariable OR of significant clinical predictors and AuROC and $95 \% \mathrm{Cl}$, without or with ICT-TB test added to the model

\begin{tabular}{|c|c|c|}
\hline \multirow{2}{*}{$\begin{array}{l}\text { Clinical } \\
\text { predictors }\end{array}$} & \multicolumn{2}{|c|}{ Multivariable OR ${ }^{a}(95 \% \mathrm{Cl})$} \\
\hline & Without ICT-TB test & With ICT-TB test \\
\hline \multicolumn{3}{|l|}{ Cough } \\
\hline$>2$ weeks versus & $4.48(4.25-4.72)$ & $3.5 \mathrm{I}(2.74-4.50)$ \\
\hline \multicolumn{3}{|l|}{$\leq 2$ weeks } \\
\hline \multicolumn{3}{|l|}{ Shaking chills } \\
\hline$\geq I$ week versus & $11.09(9.49-12.95)$ & $13.13(7.97-21.62)$ \\
\hline \multicolumn{3}{|l|}{$<$ I week } \\
\hline \multicolumn{3}{|l|}{ ARVs prescribed } \\
\hline No versus yes & I8.9| (I.4I-253.58) & $20.62(1.30-328.07)$ \\
\hline \multicolumn{3}{|l|}{$\mathrm{BMI}, \mathrm{kg} / \mathrm{m}^{2}$} \\
\hline$<19$ versus $\geq 19$ & $4.59(4.39-4.8 \mathrm{I})$ & $4.97(3.87-6.37)$ \\
\hline \multicolumn{3}{|l|}{ ICT-TB test } \\
\hline $\begin{array}{l}\text { Positive versus } \\
\text { negative }\end{array}$ & - & $5.05(0.66-38.48)$ \\
\hline AuROC (95\% Cl) & $0.90(0.85-0.95)$ & $0.92(0.88-0.95)$ \\
\hline
\end{tabular}

(from $12.5 \%$ to $27.3 \%, P=0.17$ ) and high risk groups (from $78.6 \%$ to $80.8 \%, P=0.73$ ) (Table 5).

\section{Discussion}

In January 2011, WHO reported the lack of accuracy of serological tests for TB in HIV populations; ${ }^{19}$ however, the present study is the first to assess whether addition of the ICT-TB test result extends the diagnostic value of a screening tool based on clinical predictors in Thailand. No statistically significant additional value over the four clinical factors selected was found, although the prediction probability,

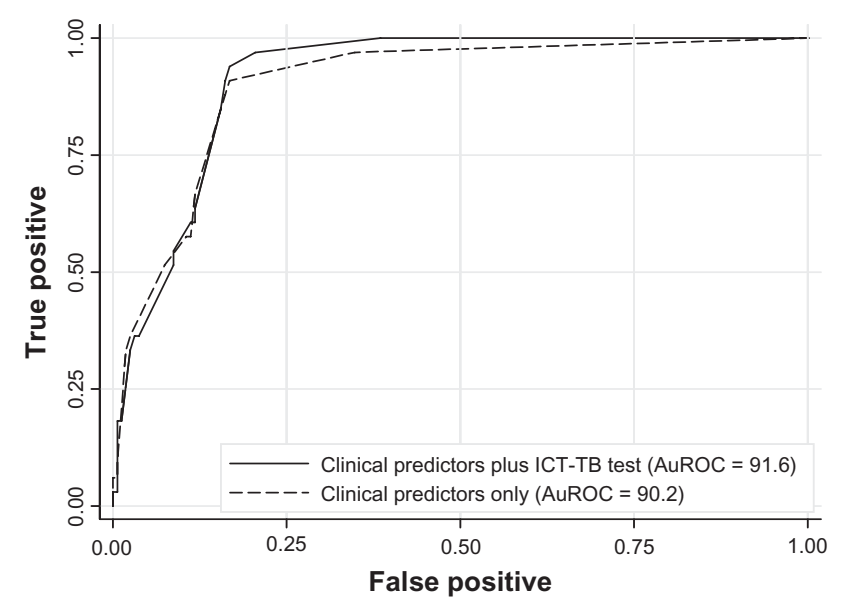

Figure 2 AuROC of four clinical predictors, with (solid line) or without (dashed line) additional ICT-TB test.

Abbreviations: AuROC, area under receiver operating curve; ICT-TB, immunochromatographic test for tuberculosis.
Table 4 Logistic coefficients, transformed scores, and assigned item scores of significant clinical predictors

\begin{tabular}{llll}
\hline $\begin{array}{l}\text { Clinical } \\
\text { predictors }\end{array}$ & Coefficient & $\begin{array}{l}\text { Transformed } \\
\text { score }\end{array}$ & $\begin{array}{l}\text { Assigned } \\
\text { score }\end{array}$ \\
\hline $\begin{array}{c}\text { Cough } \\
\leq 2 \text { weeks }\end{array}$ & 0 & 0 & 0 \\
$\quad>2$ weeks & 1.5 & 1 & 2 \\
$\begin{array}{c}\text { Shaking chills } \\
\quad \text { I week }\end{array}$ & 0 & & \\
$\geq 1$ week & 2.4 & 0 & 0 \\
ARVs prescribed & & 1.6 & 3 \\
Yes & 0 & & \\
No & 2.9 & 0 & 0 \\
BMI (kg/m² & & 1.9 & 4 \\
$\geq 19$ & 0 & 0 & 0 \\
$<19$ & 1.5 & 1 & 2 \\
\hline
\end{tabular}

Abbreviations: ARV, antiretroviral; BMI, body mass index.

demonstrated from ROC area curves, increased from 90.2\% to $91.6 \%$. NRI additionally confirmed the nonsignificant benefit of the ICT-TB test. Furthermore, we externally validated the best performing rule for TB diagnosis in HIV patients recommended by WHO, ie, presence of cough for any duration, fever, night sweats, or weight loss, in our dataset. ${ }^{10}$ The result was in accordance with the present authors' findings. AuROC without the ICT-TB was $85.5 \%$, compared with $86.3 \%$ with inclusion of the ICT-TB test result; this increase was not statistically significant $(P=0.19)$. Subgroup analysis was performed to assess any added diagnostic value in low and high TB risk groups, stratified by a clinical risk score; post-test probability of the ICT-TB did not increase significantly from pre-test probability in either subgroup, confirming a "nonbenefit" of the ICT-TB test over the screening tool in HIV patients.

The ICT-TB test used for the study is one among several commercial kits available in this country. Because antibody responses to $M$. tuberculosis antigens may be heterogeneous for a number of reasons, multiple antigens were used in the ICT-TB test to increase the performance of the test. ${ }^{15}$ The added diagnostic value may vary with the use of ICT-TB tests containing different antigens. However, the authors of this paper considered that the ICT-TB test selected for use in this study should perform best since it is a locally produced test using antigens derived from an endogenous M. tuberculosis strain and had shown higher diagnostic accuracy than an imported test. ${ }^{20}$

The combination of factors best predicting active TB in HIV patients was as follows: BMI, $<19 \mathrm{~kg} / \mathrm{m}^{2}$; cough for $>2$ weeks; shaking chills for $\geq 1$ week; and lack of ARV prescription. This is also partly consistent with predictors 
Table 5 Distribution of HIV patients with $(n=34)$ or without TB $(n=164)$ in each risk category, PPV of ICT-TB test and $95 \% \mathrm{Cl}$

\begin{tabular}{lllll}
\hline Risk category (score) & $\begin{array}{l}\text { With TB } \\
\mathbf{n}(\%)\end{array}$ & $\begin{array}{l}\text { Without TB } \\
\mathbf{n}(\%)\end{array}$ & $\begin{array}{l}\text { Prior probability (prevalence) } \\
(\mathbf{9 5 \%} \text { CI) }\end{array}$ & $\begin{array}{l}\text { PPV (95\% CI) } \\
\text { of ICT-TB }\end{array}$ \\
\hline Low risk $(\leq 7)$ & $23(12.5)$ & $161(87.5)$ & $12.5(8.1-18.2)$ & $27.3(6.0-61.0)$ \\
High risk $(>7)$ & II (78.6) & $3(21.4)$ & $78.6(4.9-95.3)$ & $80.8(28.4-99.5)$ \\
\hline
\end{tabular}

Abbreviations: $\mathrm{Cl}$, confidence interval; HIV, human immunodeficiency virus; ICT-TB, immunochromatographic test for tuberculosis; PPV, positive predictive value; TB, tuberculosis.

from other studies, ie, BMI of $\leq 18 \mathrm{~kg} / \mathrm{m}^{2},{ }^{12}$ and cough for $>2$ weeks. ${ }^{12}$ Other predictors found associated with active TB in other studies, but not in the present study, were weight loss, ${ }^{10}$ night sweats, ${ }^{10}$ fever, ${ }^{10}$ lymphadenopathy, ${ }^{11,12}$ and general weakness; ${ }^{11,12}$ these discrepant results are likely due to population differences. In some previous studies, an abnormal chest radiograph was used as one of the predictors and was proven to increase sensitivity in detection of active TB. ${ }^{10,13,14}$ However, in this study, abnormal chest radiograph was excluded as a predictor because it was used as one of the criteria for TB definition, which was likely to introduce incorporation bias.

From an economic perspective, using the ICT-TB may not seem to be particularly burdensome due to its low cost (less than US\$5). However, given the nonbenefit of the test, the Thai Ministry of Public Health budget may save more than US $\$ 8,000-10,000$ per year by solely following the screening tool developed in this study; these funds could be reallocated to subsidize other HIV-related care programs, such as TB prevention in the population at large.

Study limitations are as follows. First, M. tuberculosis culture was not used as a gold standard for diagnosis due to limited accessibility in many areas. In the present study, 14 (37.8\%) of 37 TB cases sent for M. tuberculosis culture yielded a result; of these, 13 (92.9\%) were culture-positive, reflecting a high consistence with the reference standard used in the study. Given the small number of cases with culture results, M. tuberculosis culture could not be used as a reference in regression analysis. A second limitation may be the generalizability of the study, which may be limited due to the variability of the predicting factors, prevalence of TB severity, and spectrum of the target population. Therefore, validation of the model is required before it can be adopted as routine in other health care settings. Third, some variables had missing values, such as CD 4 cell counts $(12.1 \%)$ and BMI (2.9\%). Results of multiple imputation analysis showed the same tendency, with slightly different effect sizes for each predictor. In both the original analysis and that using multiple imputation data, the ICT-TB test did not significantly add to the clinical predictors $(P=0.07)$.
During model fitting, CD4 levels were found statistically significant only in univariate analysis; however, given its clinical significance, this variable was taken into account by clustering in the final model. In future research, subgroup analysis of CD4 cell count level should be considered. Most HIV/TB patients with CD 4 cell counts less than 200 cells/ $\mu \mathrm{L}$ have low immunity and can be diagnosed with TB by routine clinical predictors (without the ICT-TB); therefore, this group may be prone to selection bias. Additionally, in a previous study, the ICT-TB test performed better when the CD4 cell count was more than 200 cells $/ \mu \mathrm{L} .{ }^{5}$ Lastly, the relatively small number of active TB patients in this study may limit the power of analysis for some variables; future research with a larger sample is warranted to confirm the results of the present study.

However, given that the present study is prospective in nature and patients were followed up for 2 months, misclassification of TB diagnosis should be minimal; this is a strength of the study.

\section{Conclusion}

In this study setting, the ICT-TB test did not improve TB diagnosis compared with the 4 clinical predictors in the overall group and any subgroups of HIV patients, as classified by clinical risk scores. Further research will be necessary to apply and compare the diagnostic performance of the clinical algorithm suggested by WHO with the clinical predictors used in this study.

\section{Acknowledgments}

The authors gratefully acknowledge participants for their contributions and Dr Weerawat Manosuthi, Andrew Mullard, and Albert Makone for assistance in preparation of the manuscript. Funding was partially provided by The Faculty of Medicine and The Graduate School, Chiang Mai University.

\section{Disclosure}

The authors declare no conflicts of interest in this research. 


\section{References}

1. World Health Organization (WHO). Global tuberculosis control: a short update to the 2009 report. Geneva, Switzerland: WHO; 2009.

2. Adjei AA, Armah H, Duah OA, Adiku T, Hesse IF. Evaluation of a rapid serological chromatographic immunoassay for the diagnosis of pulmonary tuberculosis in Accra, Ghana. Jpn J Infect Dis. 2003; 56(4):161-164.

3. Steingart KR, Henry M, Laal S, et al. A systematic review of commercial serological antibody detection tests for the diagnosis of extrapulmonary tuberculosis. Thorax. 2007;62(10):911-918.

4. Ratanasuwan W, Kreiss JK, Nolan CM, et al. Evaluation of the MycoDot test for the diagnosis of tuberculosis in HIV seropositive and seronegative patients. Int J Tuberc Lung Dis. 1997;1(3):259-264.

5. Chierakul N, Damrongchokpipat P, Chaiprasert A, Arjratanakul W. Antibody detection for the diagnosis of tuberculous pleuritis. Int $J$ Tuberc Lung Dis. 2001;5(10):968-972.

6. Gounder C, De Queiroz Mello FC, Conde MB, et al. Field evaluation of a rapid immunochromatographic test for tuberculosis. J Clin Microbiol. 2002;40(6):1989-1993.

7. Perkins MD, Conde MB, Martins M, Kritski AL. Serologic diagnosis of tuberculosis using a simple commercial multiantigen assay. Chest. 2003;123(1):107-112.

8. Talbot EA, Hay Burgess DC, Hone NM, et al. Tuberculosis serodiagnosis in a predominantly HIV-infected population of hospitalized patients with cough, Botswana, 2002. Clin Infect Dis. 2004;39(1):e1-e7.

9. World Health Organization Special Programme for Research and Training in Tropical Diseases. Laboratory-based evaluation of 19 commercially available rapid diagnostic tests for tuberculosis. Geneva, Switzerland: TDR/WHO; 2008.

10. Getahun H, Kittikraisak W, Heilig CM, et al. Development of a standardized screening rule for tuberculosis in people living with HIV in resource-constrained settings: individual participant data meta-analysis of observational studies. PLoS Med. 2011;8(1):e1000391.
11. Were W, Moore D, Ekwaru P, et al. A simple screening tool for active tuberculosis in HIV-infected adults receiving antiretroviral treatment in Uganda. Int J Tuberc Lung Dis. 2009;13(1):47-53.

12. The World Health Organization Stop TB Department. Think TB in people with HIV: start routinely screening for TB now. 2008 Mar 31. http://www.aidsmap.com/Think-TB-in-people-with-HIV-startroutinely-screening-for-TB-now/page/1443877/. Accessed August 26, 2011.

13. Cain KP, McCarthy KD, Heilig CM, et al. An algorithm for tuberculosis screening and diagnosis in people with HIV. N Engl J Med. 2010;362(8): 707-716.

14. Day JH, Charalambous S, Fielding KL, Hayes RJ, Churchyard GJ, Grant AD. Screening for tuberculosis prior to isoniazid preventive therapy among HIV-infected gold miners in South Africa. Int J Tuberc Lung Dis. 2006;10(5):523-529.

15. Verma RK, Jain A. Antibodies to mycobacterial antigens for diagnosis of tuberculosis. FEMS Immunol Med Microbiol. 2007;51(3):453-461.

16. Zucchini W. An introduction to model selection. J Math Psychol. 2000;44(1):41-61.

17. Pencina MJ, D’Agostino RB Sr, D’Agostino RB Jr, Vasan RS. Evaluating the added predictive ability of a new marker: from area under the ROC curve to reclassification and beyond. Stat Med. 2008;27(2):157-172; discussion 207-212.

18. Mohammed A, Ehrlich R, Wood R, Cilliers F, Maartens G. Screening for tuberculosis in adults with advanced HIV infection prior to preventive therapy. Int J Tuberc Lung Dis. 2004;8(6):792-795.

19. Morris K. WHO recommends against inaccurate tuberculosis tests. Lancet. 2011;377(9760):113-114.

20. Handojo I, Arifin MZ. The immunoserological diagnosis of tuberculosis: a comparison of two tests. Southeast Asian J Trop Med Public Health. 2005;36(1):141-144.
Clinical Epidemiology

\section{Publish your work in this journal}

Clinical Epidemiology is an international, peer-reviewed, open access journal focusing on disease and drug epidemiology, identification of risk factors and screening procedures to develop optimal preventative initiatives and programs. Specific topics include: diagnosis, prognosis, treatment, screening, prevention, risk factor modification, systematic

Submit your manuscript here: http://www.dovepress.com/clinical-epidemiology-journal

\section{Dovepress}

reviews, risk \& safety of medical interventions, epidemiology \& biostatical methods, evaluation of guidelines, translational medicine, health policies \& economic evaluations. The manuscript management system is completely online and includes a very quick and fair peer-review system, which is all easy to use. 